\title{
Biofumigation using a wild Brassica oleracea accession with high glucosinolate content affects beneficial soil invertebrates
}

\author{
Diana L. Zuluaga - A. E. Elaine van Ommen Kloeke • \\ Ruud Verkerk • Wilfred F. M. Röling • \\ Jacintha Ellers • Dick Roelofs • Mark G. M. Aarts
}

Received: 16 September 2014 / Accepted: 27 April 2015 / Published online: 24 May 2015

(C) The Author(s) 2015. This article is published with open access at Springerlink.com

\begin{abstract}
Aims This study explores the biofumigation effects of glucosinolate (GSL) containing Brassica oleracea plant material on beneficial, non-target soil organisms, and aims to relate those effects to differences in GSL profiles. Methods Leaf material of purple sprouting broccoli 'Santee', Savoy cabbage 'Wintessa', and the wild B. oleracea accession Winspit was analysed for GSL
\end{abstract}

Responsible Editor: John A. Kirkegaard.

Diana L. Zuluaga and A. E. Elaine van Ommen Kloeke contributed equally to this work.

D. L. Zuluaga $(\bowtie) \cdot$ M. G. M. Aarts

Laboratory of Genetics, Wageningen University,

Droevendaalsesteeg 1, 6708 PB Wageningen,

The Netherlands

e-mail: dianazuluagaa@gmail.com

A. E. E. van Ommen Kloeke · J. Ellers • D. Roelofs

Department of Ecological Science, Faculty of Earth and Life

Sciences, VU University Amsterdam, De Boelelaan 1085,

1081 HV Amsterdam, The Netherlands

R. Verkerk

Food Quality and Design, Wageningen University,

P.O. Box 17, 6700AA Wageningen, The Netherlands

\section{W. F. M. Röling}

Department of Molecular Cell Physiology, Faculty of Earth and Life Sciences, VU University Amsterdam, De Boelelaan 1085, 1081 HV Amsterdam, The Netherlands

Present Address:

D. L. Zuluaga

Istituto di Bioscienze e Biorisorse, Consiglio Nazionale delle

Ricerche, Via G. Amendola 165/A, 70126 Bari, Italy production and used for biofumigation experiments on the beneficial soil invertebrates, Folsomia candida (springtail) and Eisenia andrei (earthworm) and the soil bacterial community.

Results When mixed into soil, the Winspit plant material exerted the highest toxic effects on beneficial soil invertebrates by reducing survival and reproduction. Total GSL levels varied substantially between genotypes, in particular the aliphatic GSL (AGSL) sinigrin and gluconapin being highly abundant or exclusively present in Winspit. Differences between the genotypes regarding biofumigation effects on the soil microbial community were only observed on a temporal basis with the largest difference in bacterial community structure after 1 week.

Conclusions The high total GSL content in biofumigated soil could explain the toxicity of Winspit for soil invertebrates. These effects are likely to be the results of high AGSL levels in Winspit. The use of wild B. oleracea crops, such as Winspit, for biofumigation practices would need a proper assessment of the overall impact on soil biota before being applied on a wide scale.

Keywords Brassicaceae $\cdot$ Eisenia andrei $\cdot$ Folsomia candida $\cdot$ Gluconapin $\cdot$ Sinigrin $\cdot$ Winspit

\section{Introduction}

The current interest in glucosinolates (GSLs) mainly focuses on the toxic characteristics of GSL hydrolysis products, of which isothiocyanates (ITCs) are the best 
known (Brown and Morra 1997; Halkier and Gershenzon 2006). Isothiocyanates have, for instance, been exploited for alternative pest management methods in agriculture, called biofumigation. The release of ITCs is mediated by the enzyme myrosinase (EC 3.2.1.147) in response to tissue disruption, caused for example by herbivory (Wittstock and Halkier 2002; Bednarek and Osbourn 2009). For biofumigation, high-GSL containing Brassica plant material is mixed into the soil to suppress soil-borne pests via the release of high levels of ITCs (Matthiessen and Kirkegaard 2006). For example, reduced infestation levels of nematode cysts in potato plants can be observed due to the release of especially the aliphatic sinigrin (allyl GSL, 2-propenyl GSL) and the aromatic gluconasturtiin (2-phenylethyl GSL) (Aires et al. 2009). The toxic nature of ITCs can, however, also affect beneficial soil organisms that are essential for a proper functioning of the soil, for instance, through their effect on nutrient cycling and litter decomposition. For example, even very low concentrations of pure 2-phenylethyl ITC, spiked into the soil, reduced survival and reproduction of the beneficial soil arthropod Folsomia candida (van Ommen Kloeke et al. 2012). The effects of biofumigation on beneficial soil invertebrates using plant material, has remained largely unstudied with only a few exceptions (Vervoort et al. 2014). Such knowledge is essential for developing sustainable biofumigation strategies.

Plant species of the family Brassicaceae include commercially important crops, such as oilseed rape, cabbages, turnips and broccoli, and the scientifically important model Arabidopsis thaliana (Wittstock and Halkier 2002). More than 120 different GSLs have been identified in Brassicaceae, of which approximately $50 \%$ are aliphatic glucosinolates (AGSLs). These are methionine derived GSLs that mainly occur in leaves and flowers (Fahey et al. 2001; Halkier and Gershenzon 2006; Hopkins et al. 2009). Glucosinolate composition and content can vary widely within and between species. For instance, gluconapin (3-butenyl GSL) and glucobrassicanapin (4-Pentenyl GSL) are the most abundant GSLs in B. rapa (Padilla et al. 2007), while glucoraphanin (4-methylsulfinylbutyl GSL) is the most abundant GSL in A. thaliana (L.) Heynh, ecotype Columbia (Petersen et al. 2002), and many more examples of such variations can be found (Fahey et al. 2001). An explanation for the observed variation in GSL content and composition between species is thus the result of differences in gene expression or gene functioning of important regulators within the GSL biosynthetic pathways.

The objective of this study was to explore the effects of biofumigation on beneficial, non-target, soil organisms and the soil microbial community, using different $B$. oleracea genotypes. The observed biofumigation effects were subsequently related to differences in GSL composition. To that end, we studied the phenotypic variation for GSL production in B. oleracea. Three $B$. oleracea genotypes were chosen for this experiment, based on their expected difference in GSL content and composition. Biofumigation effects of these genotypes were assessed on the microbial (bacterial) soil community and soil invertebrate ecotoxicity models Eisenia andrei (earthworm, Oligochaeta, Lumbricidae, ecosystem engineer) and $F$. candida (fungivorous collembolan, Collembola, Isotomidae). The results of this study will aid in the development of sustainable biofumigation strategies, by predicting the consequences of biofumigation practices on keystone species of the soil ecosystem.

\section{Materials and methods}

\section{Plant material}

For all experiments three $B$. oleracea genotypes were used: Winspit (WIN), a natural wild accession originating from the coast of Dorset, England, and two cultivated F1 hybrid varieties: the purple sprouting broccoli 'Santee' (PSB) and the Savoy cabbage 'Wintessa' (SAV). Winspit is known for having high total GSL concentrations compared to other B. oleracea wild populations and cultivated genotypes (Gols et al. 2008). The two cultivated genotypes were chosen for their expected GSL content, based on information provided by the breeding company Bejo Seeds BV; where SAV was expected to have low GSL levels and PSB was expected to have high GSL levels (personal communication Dr. H. Huits). Seeds of Winspit were provided by Dr. Rieta Gols (Laboratory of Entomology, Wageningen University, the Netherlands). F1 hybrid seeds of PSB and SAV were obtained from Bejo Seeds BV (Warmenhuizen, the Netherlands).

All the seeds were sown in individual black plastic pots (17 cm diameter) containing a peat-based commercial potting mixture (Lentse potgrond nr. 4; $85 \%$ peat, $15 \%$ clay, manufactured by Lentse Potgrond, Lent, the 
Netherlands) in a temperature-controlled greenhouse at $22{ }^{\circ} \mathrm{C}$ with artificial long day light photoperiod $(16 \mathrm{~h})$.

For GSL analysis, three mature fully expanded, but not senescent, leaves were harvested after approximately eight weeks, snap frozen in liquid nitrogen and stored at $-80{ }^{\circ} \mathrm{C}$ until further analyses. Since WIN seeds were collected in the field and are likely to be genetically heterogeneous, material from four individual WIN plants was used and analysed per plant $(n=4)$ for GSL content. For both PSB and SAV a total of 12 plants were analysed, which were assumed to be genetically similar. The 12 plants were subdivided in four groups of three plants each and analysed per group $(n=4)$.

\section{Biofumigation experiments}

Biofumigation experiments were performed using the springtail Folsomia candida (Fountain and Hopkin 2005) and the earthworm Eisenia andrei, each representing a functional soil invertebrate group with important effects on soil processes (Filser 2002). $F$. candida derived from a synchronized culture of 10-12 days old animals, prepared from a laboratory stock culture ("Berlin strain", VU University Amsterdam). The International Standardization Organization (ISO) guideline 11267 (ISO 1999) was used for synchronization and stock maintenance. Springtail cultures were kept on moistened plaster of Paris mixed with charcoal and ad libitum baker's yeast as food source. E. andrei originated from a synchronized laboratory stock culture (VU University Amsterdam), cultivated in a substrate of potting soil and peat and fed abundantly with horse manure (devoid of pharmaceuticals). For the experiments, adult worms were used with a fully developed clitellum and with a maximum age difference of one month. Both worm and springtail cultures were kept under constant conditions in a climate chamber with a temperature of $20^{\circ} \mathrm{C}$, a $12 / 12 \mathrm{~h} \mathrm{light/dark} \mathrm{cycle} \mathrm{and} 75 \%$ relative humidity.

The natural soil LUFA 2.2 (Speyer, Germany) was used, which is a sandy loam soil with a pH of $5.5 \pm 0.1$ ( \pm SD) and an organic C content of $2.09 \pm 0.40 \%$. Before usage, the soil was dried at $60{ }^{\circ} \mathrm{C}$ for $24 \mathrm{~h}$ to get rid of any residual soil invertebrates.

Biofumigation was performed using freeze-dried leaves of the three different $B$. oleracea genotypes SAV, PSB and WIN. Leaves of approximately 8 weeks old were harvested from the plant, snap frozen in liquid nitrogen and then freeze-dried in order to preserve the GSL content for a longer period of time and for easy storage (personal communication Dr. John Kirkegaard). The freeze-dried plant material was stored at room temperature until used for the biofumigation experiments. For each genotype, material of several plants was combined in order to obtain enough material needed for the experiments. One percent of freeze-dried leaf material relative to total soil (on a dry weight (DW)/DW base) was used for biofumigation. Leaf material was completely fragmented using a laboratory blender (IKA, A11 basic) to ensure maximum tissue rupture and, in turn, GSL release efficiency and hydrolysis potential (Gimsing and Kirkegaard 2009).

Preparation of the experimental soil (biofumigation) was done by mixing the freeze-dried fragmented plant material thoroughly in the dry LUFA 2.2 soil by hand and kitchen blender. Subsequently, the soil was moistened to $50 \%$ of the water holding capacity of $45.2 \%$, corresponding to $22 \%$ water of the soil DW (standard ecotoxicological procedure, ISO 1999). Test animals were added immediately after the soil preparation (biofumigation) in their designated test jars (see below). Test jars were incubated in climate rooms at $20^{\circ} \mathrm{C}, 70 \%$ relative humidity and a $12 \mathrm{~h} \mathrm{light/dark} \mathrm{cycle} \mathrm{for} 28$ or 56 days, according to standard ecotoxicity conditions (ISO 1999). Once a week jars were aerated and moisture content was adjusted if needed.

The $F$. candida test consisted of six replicates per plant genotype, kept in 100-ml jars, including $15 \mathrm{~g}$ moist soil and 10 animals. After 28 days $100 \mathrm{ml}$ of water was added to each test container, stirred gently and completely poured out into a glass beaker. For each sample several digital photographs were taken to register all living springtails that were floating on the surface. To establish survival and reproduction, photos were used for hand counting the number of adult survivors and juveniles on a computer screen . The E. andrei test consisted of five replicates per plant genotype, kept in 600-ml jars and consisted of $250 \mathrm{~g}$ moist soil and five animals. After 28 days, survival was checked by hand sorting and removing the surviving adults from the soil. The remaining soil was incubated for another 28 days after which the number of hatched juveniles were extracted and counted using a water bath at $60{ }^{\circ} \mathrm{C}$.

One-way ANOVA and post-hoc tests were performed with SPSS 15.0 (SPSS Inc.) to investigate significant differences between treatments for each species. For the survival data of $F$. candida a Welch adjustment and Games-Howell post-hoc test had to be used as the assumption of homogeneity of variances was not met for 
this dataset. A Spearman Rho Correlation test was performed to investigate the correlation between average GSL content (varying per genotype used) and biofumigation effects on soil invertebrates (survival and reproduction of individual species).

Microbial community analysis

Changes in microbial community structure during and after biofumigation were determined by cultivationindependent analysis of PCR-amplified 16S rRNA gene fragments using denaturing gradient gel electrophoresis (DGGE). Samples were taken at several time points: $24 \mathrm{~h}, 48 \mathrm{~h}$, one week, two weeks and four weeks after incorporation of the plant material. Samples consisted of $250 \mathrm{mg}$ of moist soil with plant material and free of animals. Samples were frozen at $-18{ }^{\circ} \mathrm{C}$ until further analysis. DNA was extracted using the PowerSoil DNA kit (MoBio Laboratories, Solana Beach, CA) following instructions of the manufacturer. PCR-DGGE was performed as described by de Boer et al. (2012), using GCclamped primers to amplify the $\mathrm{V} 3$ region of bacterial 16S rRNA genes (Muyzer et al. 1993). The software GelCompar II (Applied Maths, Kortrijk, Belgium) was used to analyse the DGGE profiles by using a band assignment-independent method (Pearson product-moment correlation coefficient and unweighted pair-group clustering method using arithmetic averages (UPGMA). The binary matrix with Pearson correlation coefficients was analysed by nonmetric multidimensional scaling (NMDS) in PAST (PAleontological STatistics), version 2.15 (Hammer et al. 2001), which is an unsupervised statistical technique to explore similarities or distances between data points. The algorithm (Taguchi and Oono 2005) places the data in a two- or three-dimensional coordinate system to visualise the data while preserving the ranked differences and minimizing the stress (Hammer et al. 2001). For this data set the 'user similarity' was used and visualised in 2D. A stress measure smaller than 0.3 was deemed a good fit of the data.

\section{Glucosinolate analysis by HPLC}

GSLs from freeze-dried plant material $(50 \mathrm{mg})$ were extracted and subsequently analysed for GSL content and composition using high-performance liquid chromatography (HPLC), as described by Hennig et al. (2012). Benzyl GSL (glucotropaeolin, $25 \mu \mathrm{L}, 3 \mathrm{mM}$ ) was used as internal standard in the analysis.

\section{Results}

GSL analysis in B. oleracea genotypes

Total GSL concentrations of the freeze-dried material used in the biofumigation experiments was analysed using HPLC (Fig. 1). Leaves of the tested genotypes (SAV, PSB, WIN) showed large differences in aliphatic and indolic GSL composition and content. The highest total GSL content was found in the leaves of WIN, dominated by the aliphatic GSL gluconapin (3-butenyl GSL) and sinigrin (allyl GSL, 2-propenyl GSL). For PSB the most abundant GSL found was glucoiberin (3-methylsulfinylpropyl GSL), which was also the dominant GSL for SAV, showing the lowest total GSL concentrations of the $B$. oleracea genotypes.

Biofumigation effects on soil invertebrates and bacterial communities

The effects of the three different $B$. oleracea genotypes on the survival and reproduction of $F$. candida, after incorporation into LUFA 2.2 soil, were substantially different from effects on the earthworm E. andrei (Table 1). Eisenia andrei survival was unaffected by any of the three genotypes, while $F$. candida showed significant differences in survival between biofumigation treatments (Welch's, $p \leq 0.001$ ) with a significant negative correlation (Spearman's rho= $-0.894, p=0.000$ ) between average GSL total content and percentage of survival (Fig. 2a). In contrast, for both $F$. candida and $E$. andrei reproduction output was significantly different between the three genotypes ( $F=$ 200.5, $p \leq 0.001 ; F=6.06, p=0.015$, respectively). Both species showed a significant negative correlation between average GSL total content and percentage of survival (Fig. 2b) (F. candida, Spearman's rho= $-0.945, p=0.000 ; E$. andrei Spearman's rho $=-0.663$, $p=0.007$, respectively). Both $E$. andrei reproduction and $F$. candida survival and reproduction therefore corresponded to the total GSL content levels of plant material used from the three genotypes, with WIN having the highest GSL total content and negative effect, PSB intermediate and SAV the lowest (Fig. 1). WIN showed to have especially high levels of aliphatic GSL (AGSL) due to the presence of sinigrin (allyl GSL, 2porpenyl GSL) and gluconapin (3-butenyl GSL) (Fig. 1). For PSB, on the other hand, glucoiberin (3- 
Fig. 1 Glucosinolate content and composition $(\mu \mathrm{mol} / \mathrm{g})$ of freezedried, and fragmented, Brassica oleracea leaf material used for biofumigation experiments. SAV $=$ Savoy, PSB $=$ Purple Sprouting Broccoli and WIN $=$ Winspit. Error bars indicate standard errors ( $n=4$ for WIN and $n=3$ for SAV and PSB)

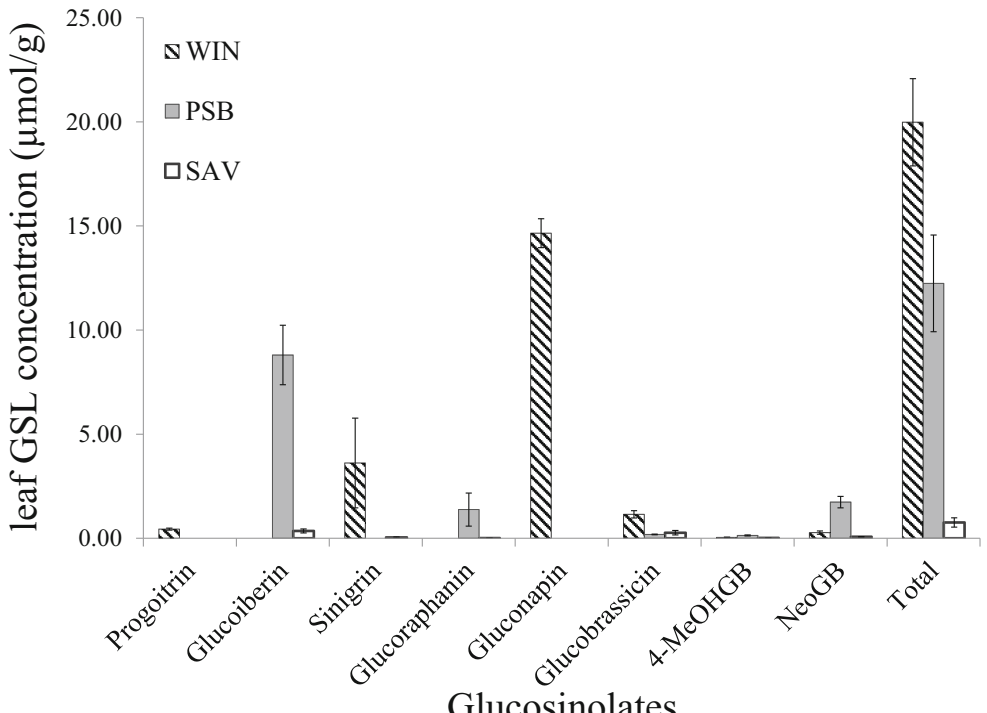

Glucosinolates methylsulfinylpropyl GSL) was the most abundant GSL, also an AGSL.

Nonmetric multidimensional scaling of soil microbial DNA revealed that effects of the $B$. oleracea plant material on the bacterial community structures did not differ between the genotypes, i.e. data points did not cluster together according to the three different genotypes on either axis (Fig. 3). Only a temporal pattern could be discovered, as the bacterial community structures were highly similar for all three genotypes across three of the four time intervals (Fig. 3). The largest difference in bacterial community structure between the genotypes was one week after biofumigation, however, after two weeks all genotypes clustered together again.

\section{Discussion}

Biofumigation experiments, jointly carried out with GSL analysis, showed that springtail survival and reproduction, as well as earthworm reproduction, were negatively correlated with total GSL content levels of plant material from the three $B$. oleracea genotypes, thus making the GSL total concentration the most likely cause of the observed changes. WIN was the genotype with the highest GSL concentrations and the genotype displaying highest toxicity to these soil invertebrates (Fig. 2). For springtails, however, experiments with single pure GSL compounds have shown that different ITCs differ greatly in their effect on survival and reproduction rates. For example, 2-phenylethyl ITC (hydrolysis product from gluconasturtiin) is approximately five times more toxic than benzyl ITC (hydrolysis product from gluconapin), when regarding the reproduction of $F$. candida and $F$. fimetaria respectively, even though these compounds differ in just one $\mathrm{CH}_{2}$-group (van Ommen Kloeke et al. 2012). In the present study, the predominant GSL in PSB was glucoiberin (3methylsulfinylpropyl GSL), while the predominant GSLs in WIN were sinigrin (allyl GSL, 2-propenyl GSL) and gluconapin (3-butenyl GSL), all aliphatic GSL (AGSL). The toxic effects of the pure ITCs corresponding to the AGSL glucoiberin, sinigrin and gluconapin on soil invertebrates are currently unknown. Therefore it remains to be tested if total GSL content or the presence of a particular AGSL in the leaf material was responsible for the observed toxic effects on the invertebrates shown here.

Interestingly, earthworm survival was not affected by the $B$. oleracea plant material. In studies with single compounds, earthworms are regularly found to be less sensitive than springtails, as for instance was found for the insecticide carbofuran (Chelinho et al. 2012) and 2phenylethyl ITC, the hydrolysis product from gluconasturtiin (van Ommen Kloeke et al. 2014). Moreover, earthworms are known to have a high tolerance towards heavy metals. This tolerance is usually interpreted as an evolutionary adaptation to their frequent burrowing through the top organic layer of soil enriched with both humus (food) and natural toxins-containing leaf litter 
Table 1 Biofumigation effects of three Brassica oleracea genotypes (WIN, PSB, SAV) on survival and reproduction of Eisenia andrei and Folsomia candida after 28 days or 56 days of exposure

\begin{tabular}{llcccccc}
\hline & & WIN & WIN-SE & PSB & PSB-SE & SAV $^{\text {SAV-SE }^{\mathrm{a}}}$ \\
\hline Survival (\%) & E.andrei & 100 & 0 & 100 & 0 & 100 & 0 \\
& F.candida & 26.7 & 7.2 & 50.0 & 5.8 & 98.3 & 1.7 \\
Reproduction (Number of juveniles) & E.andrei & 15.8 & 6.3 & 31.2 & 2.9 & 38.0 & 2.3 \\
& F.candida & 2.33 & 1.0 & 273 & 30.1 & 677 & 28.6 \\
\hline
\end{tabular}

${ }^{\text {a }} S E$ standard error with $n=5$ for $E$. andrei and $n=6$ for $F$. candida

(Morgan et al. 2007). These characteristics may also be responsible for the higher tolerance of adult earthworms towards the $B$. oleracea plant material.

Overall, the bacterial community was quite resilient to biofumigation, with a peak effect only after one week, and restoration to original values the week thereafter. In contrast, other studies observed a change in soil microbial community structures even at 3970 pmol of 2-phenylethyl ITC per gram soil (Rumberger and Marschner 2003). However, Rumberger and Marschner (2003) analysed community structure only once, after 5 days of exposure. This exposure time closely corresponds to our time point measured after one week of exposure $(+1 \mathrm{~W}$, Fig. 3), at which we found the largest differences in our study. Brown and in LUFA 2.2 soil. Exposures were conducted using $1 \%$ freezedried plant material to dry weight of soil
Morra (2009) studied the effects of soils amended with green manure (Brassicaceae crop tissue) on nitrification. The tissues with the highest GSL concentrations showed the largest inhibitory effects, although a time effect could also be observed; inhibition of nitrification was not observed at 30 days exposure and longer (Brown and Morra 2009). Our study, however, focussed on changes in the overall bacterial community and as such no conclusions can be made considering specific groups of microorganisms.

An important question to ask is whether the GSL/ITC concentrations applied in the current study are relevant for conditions in the field. Current overviews of the various biofumigation methods available, show that

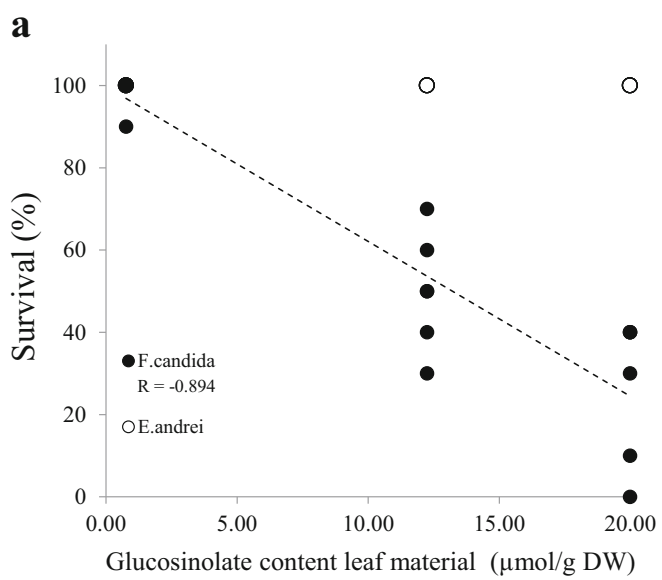

Fig. 2 Biofumigation effects of three Brassica oleracea genotypes, varying in total glucosinolate content, on a) survival and b) reproduction of beneficial soil invertebrate species, springtail Folsomia candida and earthworm Eisenia andrei, after 28 days or 56 days exposure in LUFA 2.2 soil. Exposures were conducted using $1 \%$ freeze-dried and fragmented plant material compared to

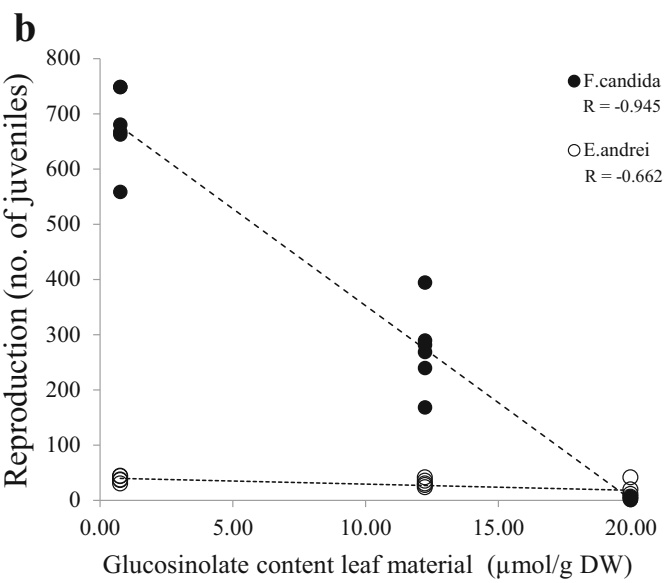

dry weight of LUFA 2.2 soil $(n=5$ for E. andrei and $n=6$ for F. candida). Lines indicate significant Spearman Rho correlations. Glucosinolate content and composition of the B. oleracea genotypes $(\mathrm{SAV}=$ Savoy, $\mathrm{PSB}=$ purple sprouting broccoli, WIN = Winspit) can be found in Fig. 1. Highest glucosinolate content was found in the WIN leaf material, followed by PSB and SAV 


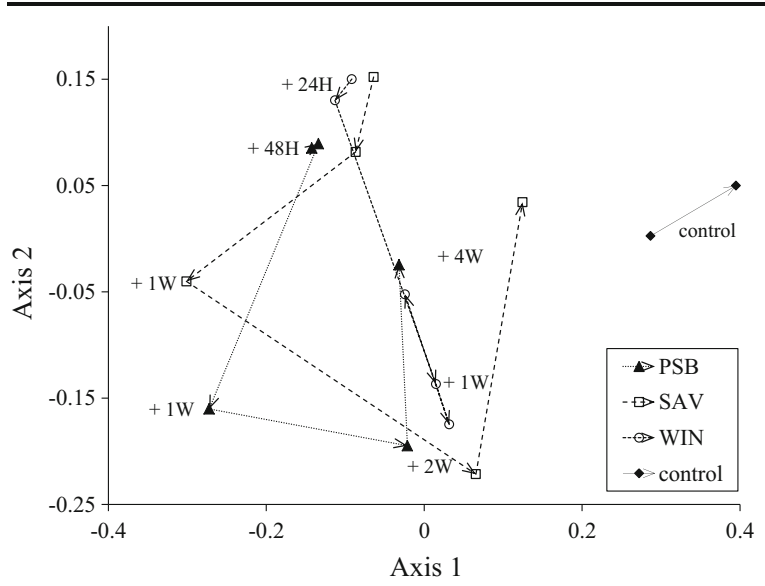

Fig. 3 Non-metric multi dimensional scaling (NMDS) data of biofumigation effects of three Brassica oleracea genotypes on the bacterial community structures at time intervals; $24 \mathrm{~h}$ ( $+24 \mathrm{~h}), 48 \mathrm{~h}(+48 \mathrm{~h}), 1$ week $(+1 \mathrm{~W}), 2$ weeks $(+2 \mathrm{~W})$ and 4 weeks $(+4 \mathrm{~W})$ exposure in LUFA 2.2 (natural) soil mixed with plant material. Exposures were conducted using $1 \%$ freeze-dried fragmented plant material compared to dry weight of LUFA 2.2 soil. SAV $=$ Savoy, PSB $=$ purple sprouting broccoli, WIN = Winspit and control $=$ LUFA 2.2. soil without treatment. Arrows indicate the sequence in time, i.e. temporal changes in bacterial community structure, exposed to a specific genotype. NMDS is an unsupervised statistical technique to visualise the distance between data points (dissimilarity matrix of Pearson correlation coefficients) (Hammer et al. 2001). This data set used 'user similarity' and $2 \mathrm{D}$ visualisation, resulting stress $2 \mathrm{D}=0.188$ (a stress measure smaller than 0.3 is deemed a good fit of the data)

during optimal biofumigation practices (maximum tissue disruption and water addition) up to $100 \mathrm{nmol}$ ITC per gram soil can be released in the soil, while a release efficacy of approximately $60 \%$ could be measured under field conditions (Gimsing and Kirkegaard 2009). In our study, the amounts potentially released were estimated to reach 88 nmol 3-butenyl ITC (derived from gluconapin, 3-butenyl GSL) per gram of soil (calculated via $1 \% \mathrm{DW}$ plant material compared to DW soil, with an average of $14.65 \mu \mathrm{mol}$ gluconapin per gram of DW plant material and assuming the same conversion efficiency). The amounts of GSL-containing plant material applied in this study are thus comparable to those found in the field when using the most optimal method available. It must be noted, however, that more commonly used biofumigation practices used in agriculture, e.g. the use of 'green manure', have much lower release efficacies (Morra and Kirkegaard 2002).

Leaves of the wild genotype WIN contained much higher concentrations of AGSL than those of the two cultivars. This is in line with previous observations by Gols et al. (2008), who compared WIN to a Brussels sprouts cultivar and to other wild B. oleracea populations, and found a lack of sinigrin in the cultivars, while it appears to be present in wild populations of B. oleracea (Mitchell and Richards 1979).

The high AGSL concentration in WIN was mainly due to the high production of gluconapin (3-butenyl GSL). This alkenyl-GSL is the direct product of the conversion of glucoraphanin (4-methylsulphinylbutyl GSL) catalysed by the 2-oxoglutarate-dependent dioxygenase. However, so far there is no scientific evidence about toxicity produced by gluconapin hydrolysis product. By contrast, several studies show a negative influence of the ITC derived from sinigrin (allyl GSL, 2propenyl GSL) for living organisms. For instance, the reduction of golden and pale potato cyst nematodes, Globodera rostochiensis appeared to be associated with high levels of sinigrin in Brassica plants (Aires et al. 2009; Lord et al. 2011). Moreover, allyl ITC (2propenyl ITC), a hydrolysis product of sinigrin, acts to suppress many species of fungi (Mayton et al. 1996; Charron and Sams 1999; Harvey et al. 2002; Troncoso et al. 2005). Therefore, it is highly possible that the negative effects caused by the wild $B$. oleracea plants to the beneficial soil invertebrates are due to their high sinigrin content.

In conclusion, our results show that $B$. oleracea plant material, enriched in particular with the AGSL sinigrin (allyl GSL, 2-proprnayl GSL) and gluconapin (3butenyl GSL), is toxic to beneficial, non-target, soil invertebrates, despite the fact that their hydrolysis products are quickly broken down in natural soil (Gimsing and Kirkegaard 2009). Especially springtails seem to suffer from elevated AGSL contents in plant material when incorporated in the soil.

A remaining challenge will be to study the effects of biofumigation under more realistic conditions of agricultural practice. For instance, our study made use of freeze-dried and completely fragmented plant material, aiming for maximal GSL hydrolysis and a so-called "worst-case scenario" (Gimsing and Kirkegaard 2009). In the field, however, plant material will likely enter the soil less fragmented and fresh. This may decrease the release of GSLs and subsequent hydrolysis into ITCs and thus moderate the biofumigation potential and effects on soil invertebrates. The results presented in this study are a first step to understand the risks associated with the introduction of plant material containing high 
levels of natural toxins in the soil and to developing sustainable biofumigation techniques.

Acknowledgments This work was funded by the research program Ecology Regarding Genetically modified Organisms (ERGO) of the Netherlands Organization of Scientific Research (NWO, project no.: 838.06.091/92).

Seeds were kindly obtained from Bejo Seeds BV (purple sprouting broccoli "Santee" and Savoy "Wintessa") and Rieta Gols (Winspit). We would like to thank Charlotte van Twisk, Irmela Kruse and Kristin Hennig for their assistance in HPLC and GCMS analysis and Corrie Hanhart for her assistance in maintaining plant material. We acknowledge Maarten Koornneef for critically reading the manuscript and his valuable comments on the work.

Open Access This article is distributed under the terms of the Creative Commons Attribution 4.0 International License (http:// creativecommons.org/licenses/by/4.0/), which permits unrestricted use, distribution, and reproduction in any medium, provided you give appropriate credit to the original author(s) and the source, provide a link to the Creative Commons license, and indicate if changes were made.

\section{References}

Aires A, Carvalho R, Conceição Barbosa M, Rosa E (2009) Suppressing potato cyst nematode, Globodera rostochiensis, with extracts of Brassicacea plants. Am J Potato Res 86:327333. doi:10.1007/s12230-009-9086-y

Bednarek P, Osbourn A (2009) Plant-microbe interactions: chemical diversity in plant defense. Science 324:746-748

Brown PD, Morra MJ (1997) Control of soil-borne plant pests using glucosinolate-containing plants. In: Agronomy DLSBT-A in (ed) Volume 61. Academic Press, pp 167-231

Brown PD, Morra MJ (2009) Brassicaceae tissues as inhibitors of nitrification in soil. J Agric Food Chem 57:7706-7711

Charron CS, Sams CE (1999) Inhibition of Pythium ultimum and Rhizoctonia solani by shredded leaves of Brassica species. J Am Soc Hortic Sci 124:462-467

Chelinho S, Lopes I, Natal-da-Luz T et al (2012) Integrated ecological risk assessment of pesticides in tropical ecosystems: a case study with carbofuran in Brazil. Environ Toxicol Chem 31:437-445

De Boer TE, Taș N, Braster M et al (2012) The influence of longterm copper contaminated agricultural soil at different $\mathrm{pH}$ levels on microbial communities and springtail transcriptional regulation. Environ Sci Technol 46:60-68

Fahey JW, Zalcmann AT, Talalay P (2001) The chemical diversity and distribution of glucosinolates and isothiocyanates among plants. Phytochemistry 56:5-51

Filser J (2002) The role of Collembolain carbon and nitrogen cycling in soil. Pedobiologia (Jena) 46:234-245

Fountain MT, Hopkin SP (2005) Folsomia candida (Collembola): a "standard" soil arthropod. Annu Rev Entomol 50:201-222

Gimsing AL, Kirkegaard JA (2009) Glucosinolates and biofumigation: fate of glucosinolates and their hydrolysis products in soil. Phytochem Rev 8:299-310
Gols R, Wagenaar R, Bukovinszky T et al (2008) Genetic variation in defense chemistry in wild cabbages affects herbivores and their endoparasitoids. Ecology 89:1616-1626

Halkier BA, Gershenzon J (2006) Biology and biochemistry of glucosinolates. Annu Rev Plant Biol 57:303-333. doi:10. 1146/annurev.arplant.57.032905.105228

Hammer Ø, Harper DAT, Ryan PD (2001) PAST: Paleontological statistics software package for education and data analysis. Palaeontol Electron 4:1-9

Harvey SG, Hannahan HN, Sams CE (2002) Indian mustard and allyl isothiocyanate inhibit Sclerotium rolfsii. J Am Soc Hortic Sci 127:27-31

Hennig K, Verkerk R, Bonnema G, Dekker M (2012) Pitfalls in the desulphation of glucosinolates in a high-throughput assay. Food Chem 134:2355-2361

Hopkins RJ, van Dam NM, van Loon JJA (2009) Role of glucosinolates in insect-plant relationships and multitrophic interactions. Annu Rev Entomol 54:57-83

ISO (1999) Inhibition of reproduction of Collembola (Folsomia candida) by soil pollutants. Switzerland, Geneva

Lord JS, Lazzeri L, Atkinson HJ, Urwin PE (2011) Biofumigation for control of pale potato cyst nematodes: Activity of Brassica leaf extracts and green manures on globodera pallida in vitro and in soil. J Agric Food Chem 59:7882-7890

Matthiessen J, Kirkegaard J (2006) Biofumigation and enhanced biodegradation: opportunity and challenge in soilborne pest and disease management. CRC Crit Rev Plant Sci 25:235265

Mayton HS, Olivier C, Vaughn S, Loria R (1996) Correlation of fungicidal activity of Brassica species with allyl isothiocyanate production in macerated leaf tissue. Phytopathology 86 : 267-271

Mitchell ND, Richards AJ (1979) Biological flora of the BritishIsles - Brassica oleracea L. J Ecol 67:1087-1096

Morgan AJ, Kille P, Sturzenbaum SR (2007) Microevolution and ecotoxicology of metals in invertebrates. Environ Sci Technol 41:1085-1096

Morra MJ, Kirkegaard JA (2002) Isothiocyanate release from soilincorporated Brassica tissues. Soil Biol Biochem 34:16831690

Muyzer G, de Waal EC, Uitterlinden AG (1993) Profiling of complex microbial populations by denaturing gradient gel electrophoresis analysis of polymerase chain reactionamplified genes coding for 16S rRNA. Appl Environ Microbiol 59:695-700

Padilla G, Cartea ME, Velasco P et al (2007) Variation of glucosinolates in vegetable crops of Brassica rapa. Phytochemistry 68:536-545

Petersen BL, Chen S, Hørslev Hansen C et al (2002) Composition and content of glucosinolates in developing Arabidopsis thaliana. Planta 214:562-571

Rumberger A, Marschner P (2003) 2-Phenylethylisothiocyanate concentration and microbial community composition in the rhizosphere of canola. Soil Biol Biochem 35:445-452

Taguchi Y-H, Oono Y (2005) Relational patterns of gene expression via non-metric multidimensional scaling analysis. Bioinformatics 21:730-740

Troncoso R, Espinoza C, Sánchez-Estrada A et al (2005) Analysis of the isothiocyanates present in cabbage leaves extract and their potential application to control Alternaria rot in bell peppers. Food Res Int 38:701-708 
Van Ommen Kloeke AEE, van Gestel CAM, Styrishave B, Hansen M, Ellers J, Roelofs D (2012) Molecular and lifehistory effects of a natural toxin on herbivorous and nontarget soil arthropods. Ecotoxicol 21:1084-1093

Van Ommen Kloeke AEE, Gong P, Ellers J, Roelofs D (2014) Effects of a natural toxin on life history and gene expression of Eisenia andrei. Environ Toxicol Chem 33:412-420
Vervoort MTW, Vonk JA, Brolsma KM, Schütze W, Quist CW, de Goede RGM, Hoffland E, Bakker J, Mulder C, Hallmann J, Helder J (2014) Release of isothiocyanates does not explain the effects of biofumigation with Indian mustard cultivars on nematode assemblages. Soil Biol Biochem 68:200-207

Wittstock U, Halkier BA (2002) Glucosinolate research in the Arabidopsis era. Trends Plant Sci 7:263-270 